\title{
Amorphous silicon based betavoltaic devices
}

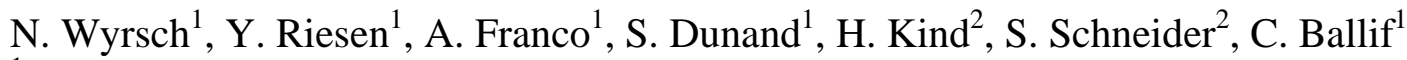 \\ ${ }^{1}$ Ecole Polytechnique Fédérale de Lausanne (EPFL), Institute of Microengineering (IMT), \\ mb-microtec, Freiburgstrasse 634, 3172 Niederwangen, Switzerland.
}

\begin{abstract}
Hydrogenated amorphous silicon betavoltaic devices are studied both by simulation and experimentally. Devices exhibiting a power density of $0.1 \mu \mathrm{W} / \mathrm{cm}^{2}$ upon Tritium exposure were fabricated. However, a significant degradation of the performance is taking place, especially during the first hours of the exposure. The degradation behavior differs from sample to sample as well as from published results in the literature. Comparisons with degradation from beta particles suggest an effect of tritium rather than a creation of defects by beta particles.
\end{abstract}

\section{INTRODUCTION}

Batteries based on radioactive sources, using betavoltaic effect, may offer advantages compared to classical chemical batteries for applications requiring very long lifetime. The betavoltaic principle is based on the direct generation of an electrical voltage and current upon irradiation of a diode by beta particles. Such a principle has been used for power generation in few satellites, but never commercialized. However, betavoltaics has recently seen a renew interest for the powering of micro-devices [1,2].

For safety reasons, it is attractive to use tritium (T) as a low energy beta particle emitter (with $5.7 \mathrm{keV}$ average energy) but it then limits considerably the power density of such a device. Such a low power is compensated by the relatively long $\mathrm{T}$ half-lifetime 12.3 years, which should allow for batteries with high energy density. Furthermore, $\mathrm{T}$ is industrially available, relatively cheap and can be embedded, if needed, in a solid matrix. Recently, a new battery design has been proposed comprising a 3D porous silicon diode to increase the surface interaction between the active volume (containing $\mathrm{T}$ ) and the semiconductor device to improve performances [3].

We here explore the possibility to use hydrogenated amorphous silicon $(\mathrm{a}-\mathrm{Si}: \mathrm{H})$ thin film device together with tritium to fabricate betavoltaic battery. In contrast to the solution incorporating a 3D porous silicon diode design, a-Si:H silicon diodes potentially offer several advantages. a-Si:H cells can be deposited on thin and (eventually) flexible substrates in order to create a 3D module design by stacking or folding such devices. They also exhibit higher performances at low excitation levels compared to c-Si diodes. A few experiments were already performed by other groups using either tritiated amorphous silicon [4] or diodes exposed to tritium [5]. In the latter case, a rapid degradation of the cell was observed, attributed to a replacement of hydrogen in a-Si:H T, leading to an increase of dangling bonds upon decay of tritium atoms into helium [6].

In the present work we will present the potential, the theoretical and practical performance of a-Si-H betavoltaic devices and analyzed the degradation of various a-Si:H diode structures exposed to tritium. Following excellent initial device performance (corresponding to the simulation), a rapid degradation is also observed which differs in some cases from the ones reported in the literature. Comparisons of this degradation from tritium exposure with the one of beta irradiation suggest that $\mathrm{T}$ rather than beta particles is involved. 


\section{SIMULATIONS}

The power density of $\mathrm{T}$ at $1 \mathrm{bar}$ is $43.8 \mu \mathrm{W} / \mathrm{cm}^{3}$. For a betavoltaic device, this power is not directly available. Beta particles emitted from the $\mathrm{T}$ should interact with a semi-conductor layer to generate e-h (electron-hole) pairs and the latter must be collected by the device electrodes. Both $\mathrm{T}$ and electrodes will also absorb beta particles leading to an attenuation of the beta flux into the device active volume. Furthermore, under the very low excitation given by the beta flux, additional loss has to be expected in the collection of carriers. In order to estimate the power output of the betavoltaic device a comprehensive simulation has been carried out.

We considered the configuration shown in Fig. 1, but neglected all lateral effects; the T layer is assumed to have a much larger spatial extension than the betavoltaic device. Simulations of the beta beam interaction with the a-Si:H based device were done using CASINO software [7]. As this software is restricted to unidirectional particle beam, absorption of betas as a function of depth was computed as a function of the angle of incidence of the incoming beam into the device. This calculation was also carried out as a function of energy as the emission of betas from $\mathrm{T}$ follows a distribution of energy with an average of $5.7 \mathrm{keV}$ and a maximum of 18 $\mathrm{keV}$. The flux of betas was then integrated for each angle and energy, taking into account the self-absorption of beta in $\mathrm{T}$ from the simplified formula [8] $\mathrm{F}=1-\mathrm{e}^{\mu \mathrm{x}}$ where $\mathrm{F}$ is the fraction of energy absorbed, $\mu$ the linear attenuation factor and $\mathrm{x}$ the travelling distance of the particle. For the simulations we used $\mu=1.81 \mathrm{~cm}-1$ and a $\mathrm{T}$ activity of $2.372 \mathrm{Ci} / \mathrm{cm}^{3}$.
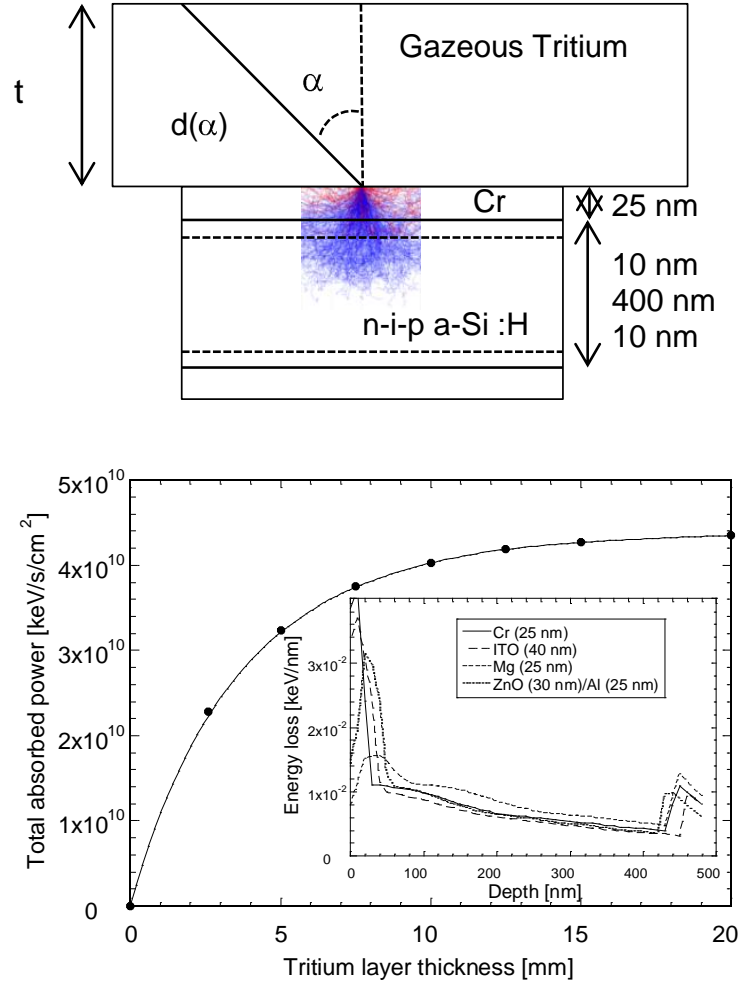

Figure 1..Configuration used for the simulations: An amorphous silicon n-i-p cell (with typically $400 \mathrm{~nm}$ thick i-layer and $10 \mathrm{~nm}$ thick doped layers) with a thin top contact (here $25 \mathrm{~nm}$ thick Cr layer) is put in contact with a layer of $\mathrm{T}$ of thickness $t$. We consider all betas hitting the cell surface coming from the apparent thickness $d$ which depends on the angle of incidence $\alpha$.

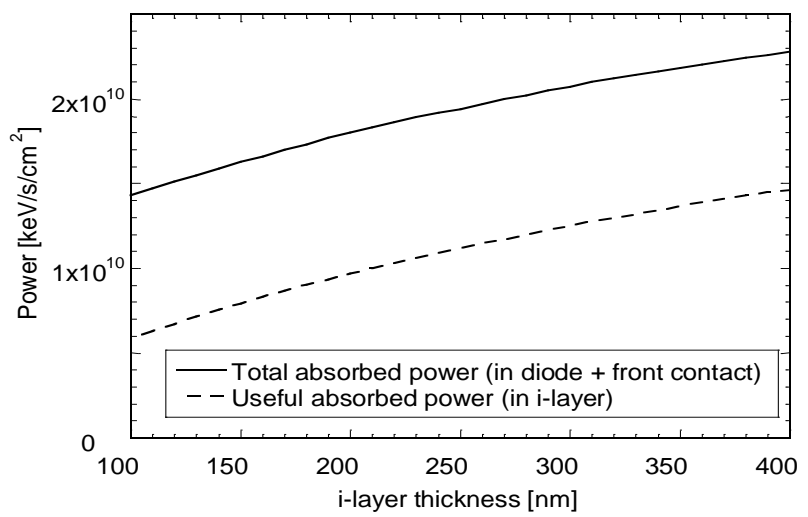

Figure 2..(left) Total power density deposited (in the diode and top contact) as a function T layer thickness in a n-i-p diode with $25 \mathrm{~nm}$ thick Cr contact, $10 \mathrm{~nm}$ thick doped layer and $400 \mathrm{~nm}$ thick i-layer, and (right) the total power density deposited in the diode and the useful power density (deposited in the diode i-layer) as a function of the intrinsic layer thickness. Energy deposited per unit depth for a single beta is shown in the inset for different top contact materials. 
Figure 2 (left) shows the power deposited in the device as a function of the T layer thickness. Due to the self-absorption of the betas by $\mathrm{T}$, the flux and the corresponding energy at the device surface saturate for $\mathrm{T}$ thickness above $5 \mathrm{~mm}$. For maximum power collection per unit volume of T, a battery configuration with two betavoltaic devices facing each other and separated by less than $3 \mathrm{~mm}$ of $\mathrm{T}$ is therefore advisable. The energy deposited as a function of depth for a single beta is also plotted in the inset for several types of top contact material and thickness. In order to minimize the absorption in top contact (which acts as a dead layer), material with low atomic number and low density should be selected. The total and useful power densities are plotted in Fig. 2 (right). The useful power density is given by the power absorbed in the i-layer.

The energy deposited by the betas lead to the generation of e-h pairs with a creation energy of ca. $4 \mathrm{eV}$ (reported values are between 3.4 and $6 \mathrm{eV}[9,10])$. The useful power of the sample of Fig. 1 corresponds, for full e-h pair collection, to a short-current $\mathrm{I}_{\mathrm{sc}}$ of $0.58 \mu \mathrm{A} / \mathrm{cm}^{2}$. Assuming, a minimum fill-factor FF of $25 \%$ and an open voltage $\mathrm{V}_{\mathrm{oc}}$ of $0.5 \mathrm{~V}$ (from $\mathrm{V}_{\mathrm{oc}}$ scaling with generation rate) a total power $\mathrm{P}$ of $0.06 \mu \mathrm{W} / \mathrm{cm}^{2}$ could be expected. Simulation of the device with ASA software [11], using e-h pair generation profile obtained from above simulations, leads to slightly lower $\mathrm{V}_{\mathrm{oc}}$ and $\mathrm{I}_{\mathrm{sc}}$ but larger $\mathrm{FF}$ and $\mathrm{P}\left(\mathrm{V}_{\mathrm{oc}}=0.36 \mathrm{~V}, \mathrm{I}_{\mathrm{sc}}=0.48 \mu \mathrm{A} / \mathrm{cm}^{2}\right.$, $\mathrm{FF}=58 \%, \mathrm{P} \approx 0.1 \mu \mathrm{W} / \mathrm{cm}^{2}$ ). Assuming that such thin diode could be deposited on a very thin substrate, with cells facing each others separated by a $2.5 \mathrm{~mm}$ layer of $\mathrm{T}$, a power density above $0.5 \mu \mathrm{W} / \mathrm{cm}^{3}$ could be achieved (with at least $8 \mathrm{~cm}^{2}$ of active area per $\mathrm{cm}^{3}$ of battery).

\section{EXPERIMENT}

Test a-Si:H diodes with an area of $0.25 \mathrm{~cm}^{2}$ were deposited on glass substrate by VHF PECVD (very high frequency plasma enhanced chemical vapor deposition) [12]. Depositions were performed at a plasma excitation frequency of $70 \mathrm{MHz}$ and at deposition temperatures of ca. $200{ }^{\circ} \mathrm{C}$. From the simulation results, two different thicknesses were chosen for the i-layer $(250$ and $400 \mathrm{~nm})$ with constant thickness $(10 \mathrm{~nm})$ for the doped layer. Various top contact materials were tested for the top contact with layer thickness between 20 and $40 \mathrm{~nm}$. These top contacts comprised $\mathrm{Al}, \mathrm{ITO}, \mathrm{ZnO}, \mathrm{ZnO} / \mathrm{Al}, \mathrm{Mg}, \mathrm{Al} / \mathrm{Mg}$ layers or layer combinations.

Prior to exposing to $\mathrm{T}, \mathrm{I}(\mathrm{V})$ characteristics of the samples were measured under white light from a sun simulator at various illumination levels using neutral density filters, down to the one corresponding to a comparable e-h pairs generation as in $\mathrm{T}$ atmosphere (ca. $0.0045 \%$ sun). For characterization in $\mathrm{T}$ atmosphere, a dedicated container was built and hooked to a $\mathrm{T}$ supply system at mb-microtec. The container was designed in such a way to have a T layer above the sample with a thickness of $2.5 \mathrm{~mm}$. I(V) characteristics were then recorded during several days using a Kethley 2612 sourcemeter. Degradation tests of diodes under electron irradiation were performed in a Philips ESEM XL30 (Environmental Scanning Electron Microscope) with a beam energy of $6 \mathrm{keV}$. The beam was intentionally defocused in order to minimize the electron-hole pair generation rate and scanned over the diode area in order to obtain uniform degradation. A total spatial generation rate of $2.7 \times 10^{15} \mathrm{~cm}^{-2}$ e-h pairs per scanned frame was estimated. Samples were scanned for up to 10 frames corresponding to ca. 3 hours of T exposure.

\section{RESULTS AND DISCUSSION}

Figure 3 exhibit the $\mathrm{I}_{\mathrm{sc}}, \mathrm{V}_{\mathrm{oc}}$ and $\mathrm{FF}$ values extracted from $\mathrm{I}(\mathrm{V})$ characteristics under white light from a solar simulator, measured on a $400 \mathrm{~nm}$ thick diode with various contacts. Ellipses 

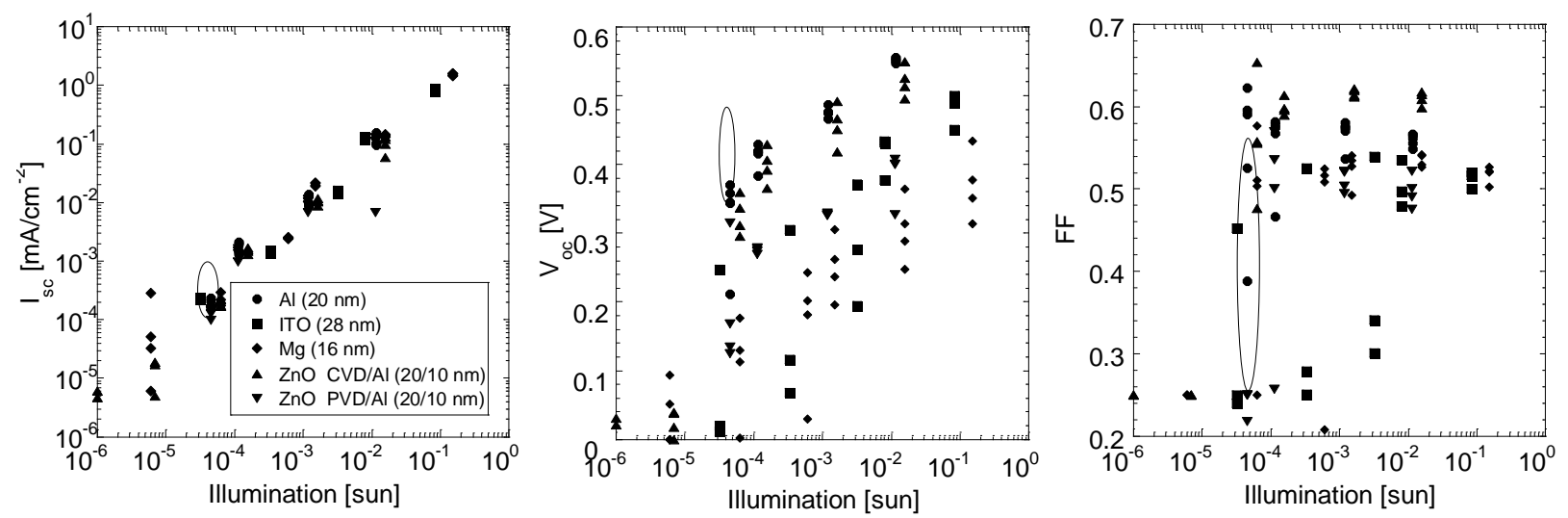

Figure 3. $\mathrm{V}_{\mathrm{oc}}, \mathrm{I}_{\mathrm{sc}}$ and $\mathrm{FF}$ as a function of light intensity of $400 \mathrm{~nm}$ thick a-Si;H diodes with various top contact. Ellipses indicate target values expected under T exposure (simulations).

indicate the expected values from the simulations. A nice match between experimental and simulated data is obtained for $\mathrm{I}_{\mathrm{sc}}$. This fact is not surprising as the equivalent target light intensity was adjusted to obtain the same generation in the diode i-layer as under T exposure. While the change of $\mathrm{V}_{\text {oc }}$ as a function of light illumination follows the same trend for all samples, the absolute values exhibit a large scattering, especially for contact deposited by sputtering (ITO and $\mathrm{ZnO}$ ). This observation seems to be linked to the small (nominal) thickness of theses layers as diode with much thicker contact layer exhibit in general much higher $V_{o c}$ values. Nevertheless, for the best diodes, $\mathrm{V}_{\mathrm{oc}}$ values close to the target ones were achieved. $\mathrm{Mg}$ contact resulted in very poor $\mathrm{V}_{\text {oc }}$ probably due to the strong degradation of the Mg layer that was observed, when deposited on a-Si:H. Some scattering is also observed in the FF values, especially at low illumination. However, part of the effect can be attributed to the evaluation procedure, as no fitting of the measured $\mathrm{I}(\mathrm{V})$ curves were performed, and the FF was deduced from the point with the maximum power. For these relatively small samples measured under very low light illumination a significant noise level induces relatively large errors in the FF determination. However, $\mathrm{FF}$ values above $50 \%$ were recorded for illumination levels equivalent to $\mathrm{T}$ exposure. From these experiments we can conclude that with $\mathrm{V}_{\text {oc }} \approx 0.35 \mathrm{~V}, \mathrm{I}_{\mathrm{sc}} \approx 0.4 \mu \mathrm{A} / \mathrm{cm}^{2}$ and $\mathrm{FF} \approx 55 \%$ a power of ca. $0.08 \mu \mathrm{W} / \mathrm{cm}^{2}$ should be attained under T exposure, as given by the simulations.

Several samples were exposed to $\mathrm{T}$ and the $\mathrm{I}(\mathrm{V})$ characteristics were recorded as a function of time. Figure 4 shows $\mathrm{I}_{\mathrm{sc}}, \mathrm{V}_{\mathrm{oc}}, \mathrm{FF}$ and power values of two $400 \mathrm{~nm}$ thick diodes with ITO contact (sample A and sample B of Fig, 3) as a function of time. I(V) characteristics of sample A just after the filling of the container with T were the following: $I_{\mathrm{sc}}=0.44 \mu \mathrm{A} / \mathrm{cm}^{2}$, $\mathrm{V}_{\mathrm{oc}}=0.49 \mathrm{~V}, \mathrm{FF}=42 \%$ and $\mathrm{P}=0.09 \mu \mathrm{W} / \mathrm{cm}^{2}$. A large and rapid degradation of $\mathrm{I}_{\mathrm{sc}}$ as well as of the $\mathrm{V}_{\mathrm{oc}}$ were observed, while the $\mathrm{FF}$ decreased and recovered. A remaining power of $10 \mathrm{nW} / \mathrm{cm}^{2}$ was measured after about $70 \mathrm{~h}$ of exposure to T. Sample B exhibits a rather different behavior. Here also $I_{s c}$ and $V_{o c}$ decrease rapidly while FF decreased but did not recovered. Fluctuations in $V_{o c}$ correlate with a variation in temperature during the experiment. Two other diodes with $\mathrm{Al}$ or $\mathrm{ZnO}$ contact layers exhibited similar behavior as sample B (as of Fig. 5). Samples A and B have a similar structure and top contact but exhibit different degradation kinetics and the origin of these differences and if contacts may influence degradation are still unknown.

The degradation behavior observed above looks at first glance similar to the one reported by Deus et al. [5], who observed a steady degradation of $\mathrm{I}_{\mathrm{sc}}$ of the diodes exposed to $\mathrm{T}$, as well as a strong initial degradation followed by a stabilization of $\mathrm{V}_{\mathrm{oc}}$ and FF. Such a behavior, was 

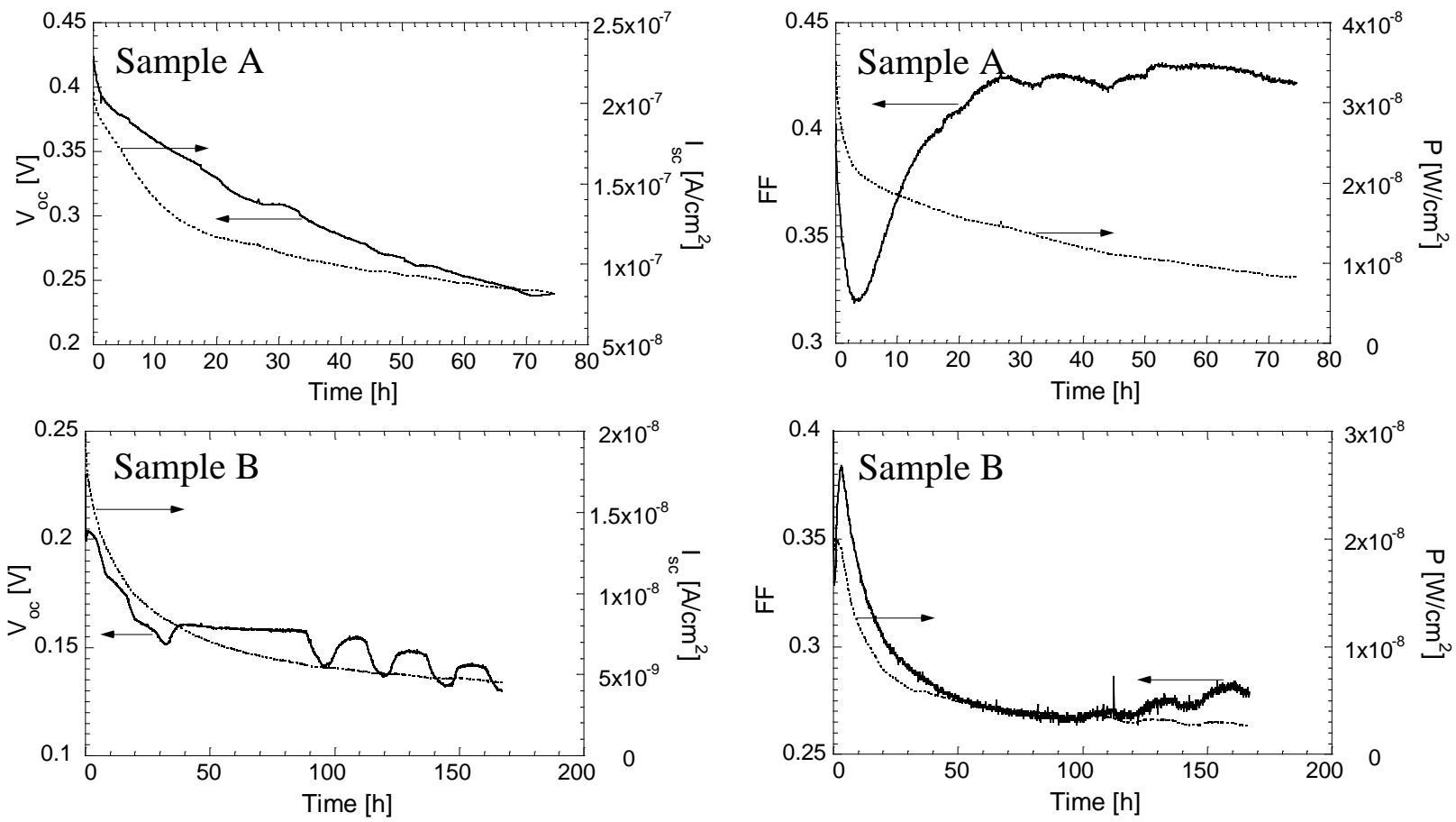

Figure 4. Power, FF, $\mathrm{I}_{\mathrm{sc}}$ and $\mathrm{V}_{\mathrm{oc}}$ of two $400 \mathrm{~nm}$ thick diodes with ITO top contact layer (sample A - top - and sample B - bottom) as a function of time under T exposure.

attributed to an exchange of $\mathrm{H}$ by $\mathrm{T}$ in the i-layer of the sample and consequently by an increase in deep defect density. However, in our case, the FF of sample A remains at a relatively high level (after the recovery phase). Such behavior, together with the decrease in $\mathrm{I}_{\mathrm{sc}}$ cannot be explained easily with a simple increase in defect density in the intrinsic layer. Measurement of the $\mathrm{I}(\mathrm{V})$ characteristics of the samples after $\mathrm{T}$ removal of the container to probe for any $\mathrm{T}$ intake by the sample remained inconclusive. In contrast, behavior of sample B, looks more similar to the one of Deus, but no conclusion can be drawn at this stage on the origin for these discrepancies and if the degradation is driven by the diffusion of $\mathrm{T}$ into the diode or by defect creation by beta particles.

In order to get more insight into the degradation mechanism, similar samples (to the ones so far studied), were characterized under low light illumination and degraded using the electron beam of the ESEM scanned over the entire surface of the diode. In order to minimize the generation density of electron-hole pairs and to improve uniformity, the beam was defocused and the experimental setup was chosen to present a significant overlap of the line scan with a low scanning speed. The total number of e-h pairs generation during the degradation experiment was adjusted to be the same as under ca. 3 hours of T exposure (biggest changes in the diode performance occurs during the first few hours, just after the beginning of T exposure). The diodes were then again characterized again under low light illumination. A very limited degradation of the performance was observed (in contrast to the ones of Fig. 4): At low light intensity roughly equivalent to $\mathrm{T}$ exposure, $\mathrm{I}_{\mathrm{sc}}$ was observed to decrease from 2.4 to $2.3 \mu \mathrm{A} / \mathrm{cm}^{2}$ while FF was reduced from 60 to $55 \%$ following the electron beam irradiation

Beta irradiations a-Si:H diodes using SEM microscopes were already performed in the past by two different groups as mean to study defect creation [13,14]. These studies using 20 $\mathrm{keV}$ beams show that a significant increase of the defect densities occurs for deposited energy well above $0.1 \mathrm{~J} / \mathrm{cm}^{2}$. In the present study the deposited energy for the beta irradiation was kept 
below this threshold, and accordingly, no degradation was expected and observed. Degradation of the diodes performance under T exposure should then linked, as suggested by Deus et al. [5], to $\mathrm{T}$ diffusion in the device structure. Due to the low $\mathrm{H}$ mobility, it is not clear how this quite rapid degradation take place in the devices.

\section{CONCLUSIONS}

Performance of a-Si:H based betavoltaic device powered by tritium have been investigated by simulation and experimentally. Such devices have the potential to deliver close to $0.1 \mu \mathrm{W} / \mathrm{cm}^{2}$ when exposed to gaseous $\mathrm{T}$ at room temperature and pressure of $1 \mathrm{~atm}$. Such power levels have been achieved experimentally just after the exposure of the devices with $\mathrm{T}$. However, a rapid degradation of the performance is observed which affects different samples differently. $\mathrm{I}_{\mathrm{sc}}$ is found to decrease considerably in all cases while $\mathrm{FF}$ and $\mathrm{V}_{\text {oc }}$ either decline or fluctuate.

The origin of the degradation and the reasons for the discrepancies are for the time being unknown. Comparisons with degradation tests from beta particles coming from an electron gun suggest that the loss in performance is related to $\mathrm{T}$ incorporated in the device. Such effect should be analyzed in details to understand the mechanism and find suitable solution to mitigate the effect. Among these solutions, additional barrier layers or new device structures could be implemented.

a-Si:H remains an attractive material for betavoltaic batteries powered by $\mathrm{T}$. The possibility to integrate serially monolithic interconnected device in the battery container would allow for a cost effective design of batteries with customized voltage output. Deposition of the betavoltaic a-Si:H device on thin substrate should enable attractive power density values without relying on 3D diode architecture.

\section{ACKNOWLEDGMENTS}

This work was supported by Swiss Commission for Technology and Innovation and by the Swiss National Science Foundation under Contract 200021_126926/1.

\section{REFERENCES}

1 L.C: Olsen et al., Physics Today 65 (2012) 35.

2. B. Liu et al., Appl. Phys. Lett. 92 (2008) 083511.

3. W. Sun et al., Adv. Mater. 17 (2005) 1230.

4. T. Kosteski et al., IEE Proc.-Circuits Devices Syst. 150 (2003) 274.

5. S. Deus, Proc. of the $28^{\text {th }}$ Photovoltaic Specialists Conf. (2000) 1246.

6. S. Zukotynski et al., J. of Non-Cryst. 299-302 (2002) 476.

7. http://www.gel.usherbrooke.ca/casino/What.html

8. DOE Handbook, DOE-HDBK-1132-99.

9. L. Hamel et al., IEEE Trans, on Nucl. Sc. 38 (1991) 251.

10. V. Perez-Mendes et al., Nucl. Instr. and Meth. in Phys. Res. A273 (1998) 127.

11. B.E. Pieters et al., Conf. Rec. of the IEEE $4^{\text {th }}$ World Conf. on PV Ener. Conv (2006) 1513.

12. N. Wyrsch et al., MRS Proc. Symp. Vol. 869 (2005) 3-14.

13. U. Schneider et al., J. of Non-Cryst. Sol. 114 (1989) 633

14. F. Diehl et al., J. of Non-Cryst. Sol. 198-200 (1996) 436 